\title{
Electric Quadrupole Coupling of the Nuclear Spin $W$ ith the Rotation of a Polar Diatomic Molecule in an External Electric Field ${ }^{1}$
}

\author{
By U. Fano
}

\begin{abstract}
Formulas are given that serve to determine the hyperfine spectrum of a rotating molecule in various cases, depending on the external field strength and on the rotational quantum number. A complete calculation is carried out for a case in which only one of the nuclei has a quadrupole moment, the molecule is in a rotational state $(1: \pm 1$ or 0 ), and the interaction between the field and the molecular dipole is comparable to that between the dipole and the nuclear spin.
\end{abstract}

\section{Introduction}

The rotation of a polar diatomic molecule in an electric field has been discussed theoretically by various authors, particularly by Brouwer [1]. ${ }^{2}$ Experimentally, this problem is being investigated at Columbia University using a modification of the standard molecular beam radio-frequency resonance method [2], in which the beam is subjected to electric instead of magnetic fields [3]. The method permits the energy differences between rotational states of the molecule to be measured. Various spin-dependent interactions between the nuclei and the rest of the molecule may give rise to a hyperfine structure of the rotational spectrum. The electric quadrupole interaction between the nuclei and the molecular electrons, if present at all, is likely to be the major factor in this connection and is the subject of the present theoretical investigation.

Molecular rotation. - The qualitative aspects of molecular rotation in an electric field will be reviewed here, disregarding the effects that give rise to a hyperfine spectrum. The electronic system of the molecule is assumed to be in a ${ }^{1} \Sigma$ state, so that it has no net angular momentum. The rotation of the molecule is then similar to that of a dumbbell whose two spheres (the two

\footnotetext{
${ }_{1}$ Part of this work was performed at the Pupin Physics Laboratory, Columbia University, during the tenure of a Research Associateship of the Carnegie Institution of Washington in 1946.

${ }^{2}$ Figures in brackets indicate the literature references at the end of this paper.
}

atoms) carry charges of opposite sign. Several cases may be considered:

(a) The electric field is so strong that the potential energy of the molecule, when oriented along the field, is much greater than its rotational kinetic energy. Then the molecule remains oriented along the field, except for minor oscillations about this position. This case is unrealistic, however, as no sufficiently strong field is currently available.

(b) The field is vanishingly weak, so that the molecule rotates freely about its center of mass with a constant, quantized, angular momentum. The component of this angular momentum along the field is then also constant and quantized. The positively charged part of the molecule points in the direction of the field about as frequently as in the opposite direction. Thus the molecular electric moment averages out, and the presence of the field has no effect on the rotational energy levels.

(c) The field is moderately weak. The rotation is now appreciably perturbed by the field, and the molecular electric moment no longer averages out; an electric polarization is thus induced and each rotational energy level is accordingly lowered by an amount equal to a polarization coefficient times the square of the electric field. When the rotation has primarily the character of a precession (clockwise or counterclockwise) around the direction of the field, the effect of the field is to force the positively charged part of the molecule to lean in 
the direction of the field, and so induces a positive polarization. An opposite effect occurs, however, when the rotation brings the positively charged part alternately close to the direction of the field (downhill), and then away from it in the opposite direction (uphill); the positive charge moves rapidly while it is downhill and spends little time there, but it lags in the uphill position, and on the average it will spend more time uphill, giving rise to a negative polarization.

(d) The field is intermediate or moderately strong. The rotation is perturbed by the field to a greater and greater extent. As the field increases, the positively charged part of the molecule is forced to lean in the direction of the field, whatever the type of rotation may be, and the polarization is always positive.

The quantitative treatment of cases (a), (b), and (c) is simple, but in case (d), which obtains most frequently, the energy levels cannot be given in general as analy tic functions of the electric field. In work as yet unpublished, W. E. Lamb, Jr., has developed a convenient method for the numerical computation of the terms for any value of the field strength. The rotational wave equation is

$$
\left[-\left(\dot{\pi}^{2} / 2 A\right) \Delta-l E \cos \vartheta_{\boldsymbol{R}}-W\right] \psi\left(\vartheta_{\boldsymbol{R}}, \varphi_{\boldsymbol{R}}\right)=0,
$$

where the electric field, $\boldsymbol{E}$, is taken as a polar axis, $\left({ }^{2}, \varphi_{\boldsymbol{R}}\right)$ are polar coordinates of a vector $\boldsymbol{R}$ joining the nuclei, $l$ is the dipole moment of the molecule $A$ its moment of inertia, $\Delta$ the two-dimensional Laplace operator (in the coordinates $\vartheta_{\boldsymbol{R}}, \varphi_{\boldsymbol{R}}$ ), and $W$ the energy eigenvalue. The first term in brackets represents the kinetic energy of rotation; the second represents the potential energy of the dipole in the electric field. A dimensionless form is obtained by dividing the equation by $\left(-\hbar^{2} / 2 A\right)$ :

$$
\left(\Delta+\alpha \cos \vartheta_{\boldsymbol{R}}+w\right) \psi=0,
$$

where $\alpha=l E /\left(\hbar^{2} / 2 A\right)$ is a numerical index of the field strength and $w=W /\left(\hbar^{2} / 2 A\right)$. The eigenfunctions can be labeled by means of two quantum numbers $n$ and $m ; m$ is the magnetic quantum number, which indicates the rate and the direction of the precession of the molecule around the electric field; $n-|m|$ is the number of nodal lines, which characterizes the component of the motion along the meridians of the polar coordinates. The eigenvalues are functions of $\alpha$. For all values of $\alpha$, we have $w_{n, m}=w_{n,-m}$, as the energy does not depend on the direction of the precession. For $\alpha=0$, the results are familiar, namely, $v_{n, m}=$ $n(n+1), \psi_{n, m}$ is a spherical harmonic, and $n$ is an azimuthal quantum number that measures the total angular momentum. For $\alpha \ll 1$, case $(c)$, a perturbation calculation gives $w_{n m} \sim n(n+1)+$ $\alpha^{2}[(n+m)(n-m) / 2 n(2 n-1)(2 n+1)-(n+1+m)$ $(n+1-m) / 2(n+1)(2 n+1)(2 n+3)]$; as expected, the coefficient of $\alpha^{2}$ is positive for $|m / n| \sim 1$ (fast precession), negative for $|m / n| \ll 1$.

Quadrupole interaction.-The electrical charge distribution of a nucleus having a quadrupole moment resembles an ellipsoid of rotation rather than a sphere (the direction of the nuclear spin being the axis of symmetry of the ellipsoid). The value and sign of the quadrupole moment indicate the elongation of the ellipsoid. As the charge distribution of a diatomic molecule is not spherically symmetrical about any of its nuclei, the electrostatic energy of the nucleus within the molecule will depend upon the orientation of the nuclear spin with respect to the axis of symmetry of the molecule (i. e., the line joining the nuclei). If the spin be directed along the axis and then swung into the opposite direction and back into the initial one, the interaction energy will go through two maxima and two minima.

In the absence of this quadrupole interaction and of magnetic interaction, the nuclear spins would maintain a constant orientation in space while the polar molecule rotates in an electric field, and the orientation of each nuclear spin with respect to the axis of the rotating molecule would undergo periodic variations. In the presence of a quadrupole moment there will then be periodic variations of the interaction, and hence torques that affect both the rotation of the molecule and the orientation of the nuclear spins. This effect will be particularly conspicuous in case (b) described above, as the orientation of the angular momentum of the molecular rotation can then be shifted by a torque without perfor ming any work. This angular momentum will then precess freely together with the nuclear spins, and only the total angular momentum, i. e., the vector sum of the molecular momentum and of the nuclear spins, will remain constant. When, on the other hand, considerable work must be performed to upset the molecular rotation, the quadrupole interaction will have no significant effect on the motion of the 
molecular constituents; the energy of the system will then simply include a contribution from the quadrupole interactions averaged over the varying orientation of the spins with respect to the molecular axis. This contribution will depend on the state of rotational motion of the molecule and on the (constant) orientation of the nuclear spins with respect to the electric field. This situation prevails generally in cases (a) and (d) described above, i. e., for moderately to very strong fields; from this standpoint, case (c) represents an intermediate situation.

It should still be considered that no work is ever required for the simple operation of reversing the direction of the precession of the molecule around the electric field, provided the speed of precession is unchanged. Owing to the quadrupole interaction, and for any electric field strength, the uniform precession might therefore conceivably be turned into a precession whose direction is periodically reversed; the conservation of angular momentum would be preserved by corresponding variations of the precession of the nuclear spins. Quantum mechanically, there would thus be "standing waves of precession," the number of nodes of the standing wave depending on the speed of precession. However, it will be shown further below that only the standing wave with two nodes actually arises in this way. This is a direct consequence of the particular dependence of the quadrupole interaction upon the mutual orientation of the molecule and of the nuclear spin; as already stated this interaction goes through two maxima and minima when a cyclic change of orientation is performed.

The quantitative treatment of our problem will accordingly proceed as follows. As shown by Casimir, [4], the interaction energy between a nuclear quadrupole moment and the electronic charge of an atom or molecule is of the order of $e^{2} q Q / 4 \quad(q$ and $Q$ are the usual symbols for a molecular and a nuclear constant whose product is $\left\langle 1 \mathrm{~cm}^{-1}\right)$. Hence, taking into account the possible values of the pertinent constants, it seems safe to assume for the dimensionless parameter characterizing this interaction:

$$
|\beta|=\left|e^{2} q Q / 4\left(\hbar^{2} / 2 A\right)\right| \lesssim 10^{-2}
$$

This value must be related to the fact that, in general, the separation of different unperturbed rota- tional levels of the molecule is ${ }^{3}\left|w_{n^{\prime} m^{\prime}}-w_{n m}\right| \geq 1$. Therefore, in general, the quadrupole interaction can be treated as a small perturbation of each rotational term, and each term will preserve its quantum numbers $n, m$. This procedure will not be adequate in the cases discussed above, in which the quadrupole interaction has a great effect on the rotation of the molecule, namely, for states $(n, m)$ which are directly coupled by the quadrupole interaction to other states $\left(n^{\prime}, m^{\prime}\right)$ such that $\left|w_{n^{\prime} m^{\prime}}-w_{n m}\right| \leq|\beta|$. Direct quadrupole coupling means that the quadrupole interaction energy $H_{Q}$ has a matrix element $\left(n^{\prime} m^{\prime}\left|H_{Q}\right| n m\right) \neq 0$; this occurs only when $\left|m-m^{\prime}\right| \leqslant 2$, as it will be shown in the next section. These two conditions can be fulfilled simultaneously in two cases only:

(1) $n=n^{\prime}>0, m=-m^{\prime}= \pm 1$ (all values of $\alpha$ ), in which the molecule precesses back and forth, i. e., clock- and counterclockwise;

(2) $n=n^{\prime}>0, \alpha^{2} \leq|\beta|\left(\left|m-m^{\prime}\right| \leqslant 2\right)$, in which the field is weak and the rotation is strongly perturbed.

The latter case becomes simpler when $\alpha^{2} \ll|\beta|$ or even $\alpha^{2}=0$. In fact, the zero-field case involves a simple vector coupling of the rotational angular momentum $\boldsymbol{J}(\boldsymbol{J}=n)$ with the nuclear spins $\boldsymbol{I}_{1}$ and $\boldsymbol{I}_{2}$. In the case of near-zero-field, this vector coupling is preserved and the effect of the electric field can be treated as a small perturbation, somewhat analogous to the anomalous Zeeman effect produced by a weak magnetic field.

The general expression for the quadrupole interaction matrix is given in the next section; the application to the different cases will then follow.

\section{Matrix Elements of Quadrupole Inter- action Between Molecular Rotation and a Spinning Nucleus}

(a) The electrostatic interaction $-e^{2} /|\boldsymbol{r}-\boldsymbol{p}|$, between a nuclear proton at a position $\boldsymbol{p}$ with respect to the center of the nucleus and a molecular electron at a position $\boldsymbol{r}$ with respect to the same center, can be expanded into a series of Legendre spherical functions of the angle $\gamma_{r p}$ between the vectors $\boldsymbol{r}$ and $\boldsymbol{p}$. The first term of this series

\footnotetext{
${ }^{3}$ The magnetic spin-spin interaction between the nuclei and the interaction between the nuclear magnetic moments and the rotation-induced magnetic moment of the molecule are generally weaker than the electric quadrupole interaction; in our scale they may be characterized by a parameter $\eta \leq 10^{-5}$. These interactions will be disregarded in the following therefore we must assume

$$
|n| \ll|\beta| \leq 10^{-2}, B^{2} \sim 0 .
$$
}


represents the Coulomb attraction of the electron toward the center of the nucleus, the second term has no average effect, and the third, namely:

$$
\begin{aligned}
& H_{Q}=-e^{2}\left(p^{2} / r^{3}\right) P_{2}\left(\cos \gamma_{\boldsymbol{p r}}\right)= \\
& -e^{2}\left(p^{2} / r^{3}\right)\left(\frac{3}{2} \cos ^{2} \gamma_{\boldsymbol{p r}}-\frac{1}{2}\right)
\end{aligned}
$$

is called the quadrupole component of the interaction. This component must be integrated over the density of protons throughout the nucleus and the density of electrons throughout the molecule. The integration over the electron density is carried out in polar coordinates having as axis a vector $\boldsymbol{R}$ from the center of the molecule to the center of the nucleus. Using the addition theorem of spherical harmonics and taking into account the fact that the $\Sigma$-type electronic state has axial symmetry, we find:

$$
\begin{aligned}
& \left\langle\left(1 / r^{3}\right) P_{2}\left(\cos \gamma_{\boldsymbol{p} r}\right)\right\rangle_{\boldsymbol{r}}= \\
& \left\langle\left(1 / r^{3}\right) P_{2}\left(\cos \gamma_{\boldsymbol{R} r}\right)\right\rangle_{\boldsymbol{r}} P_{2}\left(\cos \gamma_{\boldsymbol{R} \boldsymbol{p}}\right),
\end{aligned}
$$

where the symbol \langle\rangle$_{r}$ indicates integration with respect to the electron density. The first factor on the right-hand side is a molecular characteristic which is indicated [4] by $q / 2$. Using again the addition theorem of spherical harmonics,

$$
\begin{aligned}
& P_{2}\left(\cos \gamma_{\boldsymbol{R} \boldsymbol{p}}\right)= \\
& (4 \pi / 5) \Sigma_{\mu}(-1)^{\mu} Y_{2,-\mu}\left(\vartheta_{\boldsymbol{p}}, \varphi_{\boldsymbol{p}}\right) Y_{2, \mu}\left(\vartheta_{\boldsymbol{R}}, \varphi_{\boldsymbol{R}}\right),
\end{aligned}
$$

we have,

$$
\begin{aligned}
& \left\langle H_{Q}\right\rangle_{r}= \\
& \left(-e^{2} q / 2\right) \Sigma_{\mu}(-1)^{\mu}\left[p^{2}(4 \pi / 5)^{1 / 2} Y_{2,-\mu}\left(\vartheta_{\boldsymbol{p}}, \varphi_{\boldsymbol{p}}\right)\right] \\
& \times\left[(4 \pi / 5)^{1 / 2} Y_{2, \mu}\left(\vartheta_{\boldsymbol{R}}, \varphi_{\boldsymbol{R}}\right)\right] .
\end{aligned}
$$

(The normalization of the spherical harmonics is such that $\int \mathcal{S}\left|Y_{l, \mu}\right|^{2} \sin \vartheta d \vartheta d \varphi=1, Y_{l, \mu}^{*}=(-1)^{\mu} Y_{l,-\mu}$.

(b) All the matrix elements of the first factor in square brackets, i. e., the integrals of this factor over the proton density, associated with pairs of nuclear states with the same spin $I$ and magnetic quantum numbers $m_{I}, m_{I}{ }^{\prime}$, can be given in terms of just one such matrix element. Thus:

$$
\begin{aligned}
& \left(m_{I}{ }^{\prime}\left|p^{2}(4 \pi / 5)^{1 / 2} Y_{2,-\mu}\left(\vartheta_{\boldsymbol{p}}, \varphi_{\boldsymbol{p}}\right)\right| m_{I}\right)= \\
& \left(I\left|p^{2}(4 \pi / 5)^{1 / 2} Y_{2,6}\left(\vartheta_{\boldsymbol{p}}, \varphi_{\boldsymbol{p}}\right)\right| I\right)\left(D_{I m_{I}}^{I m_{I}^{\prime}} / D_{I I}^{I I}\right) \delta_{m_{I^{\prime}}, m_{I^{-}}} .
\end{aligned}
$$

The first factor on the right-hand side is a nuclear characteristic which is indicated [4] as $Q / 2$ (i. e., half of the quadrupole moment). In the formula $\delta_{i, k}=1$ or 0 , depending on whether $i=k$ or $i \neq k$. The coefficients $D_{j m}^{j^{\prime} m^{\prime}}$ may be found by means of group theory, ${ }^{4}$ and are given in table 1. As $D_{I I}^{I I}=-I(2 I-1) /(2 I-1)(2 I+3)$ we take:

$$
d(j)_{m}^{m^{\prime}}=-(2 j-1)(2 j+3) D_{i m}^{i m^{\prime}} ;
$$

the coefficients $d$ are given in table 2 . Thus:

$$
\begin{aligned}
& \left(m_{I}-\mu\left|p^{2}(4 \pi / 5)^{1 / 2} Y_{2, \mu}\left(\vartheta_{\boldsymbol{p}}, \varphi_{\boldsymbol{p}}\right)\right| m_{I}\right)= \\
& Q d(I)_{m_{I}}^{m_{I}-\mu} / 2 I(2 I-1) .
\end{aligned}
$$

TABLE 1.-Coefficients $D_{j, m}^{i^{\prime}, m^{\prime}}$ for: $j^{\prime}-j=0, \pm 2$; $m^{\prime}-m=0$, $\pm 1, \pm 2 .^{a}$

$$
\begin{aligned}
D_{j, m}^{i, m}=- & {\left[3 m^{2}-j(j+1)\right] /(2 j-1)(2 j+3) } \\
D_{j, m}^{j, m+1}= & (2 m+1)[(3 / 2)(j+m+1)(j-m)]^{1 / 2} \\
D_{j, m}^{i, m+2}=- & {[(3 / 2)(j+m+2)(j+m+1)(j-m)(j-m-1)]^{1 / 2} /(2 j-1)(2 j+3) } \\
D_{j, m}^{i+2, m}= & (3 / 2)\left[(j+m+2)(j+m+1)(j-m+2)(j-m+1) /(2 j+1)(2 j+3)^{2}\right. \\
& (2 j+5)]^{1 / 2} \\
D_{i, m}^{j+2, m+1}= & {\left[(3 / 2)(j+m+3)(j+m+2)(j+m+1)(j-m+1) /(2 j+1)(2 j+3)^{2}\right.} \\
& (2 j+5)]^{1 / 2} \\
D_{j, m}^{j+2, m+2}= & {\left[(3 / 8)(j+m+4)(j+m+3)(j+m+2)(j+m+1) /(2 j+1)(2 j+3)^{2}\right.} \\
& (2 j+5)]^{1 / 2}
\end{aligned}
$$

a All other coefficients are obtained by means of the formulas

$$
D_{i^{\prime} m^{\prime}}^{i m}=(-1) m^{\prime-m} D_{j m}^{i^{\prime \prime} m^{\prime}}, D_{i,-m}^{i^{\prime},-m^{\prime}}=D_{i m}^{i^{\prime \prime} m^{\prime}}
$$

TABLE 2.-Coefficients $d(j)_{m}^{m^{\prime}}=-(2 j-1)(2 j+3) D_{j m}^{j m^{\prime}}$

$$
\begin{aligned}
& d_{m}^{m+2}=[(3 / 2)(j+m+2)(j+m+1)(j-m)(j-m-1)]^{1 / 2} \\
& d_{m}^{m+1}=-(2 m+1)[(3 / 2)(j+m+1)(j-m)]^{1 / 2} \\
& d_{m}^{m}=3 m^{2}-j(j+1) \\
& d_{m}^{m-1}=(2 m-1)[(3 / 2)(j-m+1)(j+m)]^{1 / 2}=d_{-m}^{-m+1} \\
& \left.d_{m}^{m-2}=!(3 / 2)(j-m+2)(j-m+1)(j+m)(j+m-1)\right]^{1 / 2}=d_{-m}^{-m+2}
\end{aligned}
$$

(c) The matrix elements of the factor in the second bracket of eq $3 \mathrm{a}$, pertaining to the rotational states of the molecule $(n, m)$ and $\left(n^{\prime}, m^{\prime}\right)$ is the average of this factor over the probability distribution $\psi_{n^{\prime}, m^{\prime}}^{*} \psi_{n, m}$ and can be indicated as

\footnotetext{
${ }_{4}$ These coefficients are closely related to those indicated by $\mathrm{E}$. Wigner, Gruppentheorie (Vieweg, Braunschweig 1931), p. 206, as $S_{j / m \mu}^{(j 2)}$. For $j$ and $j^{\prime}$ integer $D_{j m}^{j^{\prime} m^{\prime}}=(4 \pi / 5)^{1 / 2} \mathcal{\int} \mathcal{\int} Y^{*}{ }_{j^{\prime},-m^{\prime}}(\vartheta \varphi) Y_{2, m^{\prime}-m}(\vartheta, \varphi) Y_{j, m}(\vartheta \varphi) \sin \vartheta d \vartheta d \varphi$. The formula given means essentially that the ratio of matrix elements for different values of $\left(m_{I}, m_{I}^{\prime}\right)$ is the same as though the angular distribution of the proton density in each nuclear state $\left(I, m_{I}\right)$ were described by the spherical harmonic $Y_{I}, m_{I}$ (suitably generalized for half-integer $I$ ).
} 


$$
\begin{aligned}
\left.n^{\prime}, m^{\prime}\left|(4 \pi / 5)^{1 / 2} Y_{2 \mu}\left(\theta_{\boldsymbol{R}}, \varphi_{\boldsymbol{R}}\right)\right| n, m\right) & =(4 \pi / 5)^{1 / 2} \mathcal{S} \int \psi_{n^{\prime}, m^{\prime}}^{*}\left(\theta_{\boldsymbol{K}} \varphi_{2:}\right) Y_{2, \mu}\left(\theta_{\boldsymbol{R}}, \varphi_{\boldsymbol{R}}\right) \psi_{n, m}\left(\theta_{\boldsymbol{R}}, \varphi_{\boldsymbol{R}}\right) \sin \theta_{\boldsymbol{R}} d \theta_{\boldsymbol{R}} d \varphi: \\
& =R_{n, m}^{n^{\prime}, m^{\prime} \delta_{m^{\prime}}, m+\mu .}
\end{aligned}
$$

Expanding the rotational wave functions $\psi_{n, m}$ into spherical harmonics:

$$
\psi_{n, m}(\theta, \varphi)=\Sigma_{\mathbf{J}} C_{J}^{n, m} Y_{\mathbf{J}, m}(\theta, \varphi),
$$

where the coefficients $C_{J}^{n m}$ depend on the parameter $\alpha$, we have:

$$
\begin{aligned}
R_{n, m}^{n^{\prime}, m^{\prime}}= & \Sigma_{J}\left(D_{J, m}^{J+2, m^{\prime}} C_{J+2}^{n^{\prime} m^{\prime}}+D_{J, m}^{J m^{\prime}} C_{J}^{n^{\prime} m^{\prime}}+\right. \\
& \left.D_{J, m}^{J-2, m^{\prime}} C_{J-2}^{n^{\prime} m^{\prime}}\right) C_{J}^{n m} .
\end{aligned}
$$

For $n=n^{\prime}, \quad m=m^{\prime}$ (which is the only case involved in a first-order perturbation), we have:

$R_{n, m}^{n, m}=R(n, m)=(3 / 2)\left\langle\cos ^{2} \theta\right\rangle_{\boldsymbol{R}}-1 / 2$.

There is also a simpler form for

$$
R_{n, 1}^{n,-1}=S(n)=(3 / 8)^{1 / 2}\left\langle\sin ^{2} \theta \boldsymbol{R}\right\rangle .
$$

The coefficients $R$ must be obtained by numerical computation except when $\alpha$ is very large or very small. For $\alpha \ll 1$ :

$C_{J}^{n, m}=\delta_{n, J}+\alpha \delta_{n \pm 1, J} f(n, n \pm 1)+$ terms in $\alpha^{2}$, etc., [1]

where $f$ is a function of $n$ and $n \pm 1$; hence eq 5 a gives

$$
R_{n, m}^{n \cdot m^{\prime}}=D_{n, m}^{n \cdot m^{\prime}}+\text { terms in } \alpha^{2} \text {, etc. }
$$

For $\alpha \gg 1,\left|\psi_{n, m}\right| \sim 0$, except for $\vartheta_{\mathbf{R}} \ll 1$ (i. e., the molecule points approximately in the direction of the field). Hence, according to eq $5, \lim _{\alpha=\infty} R_{n, m}^{n^{\prime}, m^{\prime}}=$ $\delta_{n, n^{\prime}} \delta_{m, m^{\prime}}$. However, the convergence to this limit is slow. For example, $R(n, m)$ can be expanded in powers of $\alpha^{-1 / 2}$ as follows:

$$
\begin{aligned}
& R(n, m)= \\
& 1-3(2 n-m+1)(2 \alpha)^{-1 / 2}+\text { terms in } \alpha^{-1}, \text { etc. }
\end{aligned}
$$

(d) Combining eq 3a, 4, and 5, the matrix element of $H_{Q}$, pertaining to states with the same total magnetic quantum number $M=m_{1}+m=$ $m^{\prime}{ }_{1}+m^{\prime}$, is found to be

$$
\begin{aligned}
& \left(n^{\prime}, m^{\prime}\left|H_{Q}\right| n, m\right)= \\
& -\left(e^{2} q Q / 4\right)(-1)^{m \prime-m} R_{n, m}^{n^{\prime}, m^{\prime}} d(I)_{M-m}^{M-m^{\prime}} / I(2 I-1)= \\
& -\left(\hbar^{2} / 2 A\right)(-1)^{m-m^{\prime}} \beta^{\prime} R_{n, m}^{n^{\prime}, m^{\prime}} d(I)_{M-m}^{M-m^{\prime}},
\end{aligned}
$$

where

$$
\beta^{\prime}=\beta / I(2 I-1)=\left[e^{2} q Q / 4 I(2 I-1)\right] /\left(\hbar^{2} / 2 A\right) .
$$

\section{The General Case: $\alpha^{2} \gg|\beta|,|m| \neq 1$}

Here it is sufficient to treat the quadrupole coupling as a first order perturbation of each rotational term.

Only one of the nuclei has a quadrupole moment.Each term of the complete system is characterized by the three quantum numbers $n, m$, and $m_{I}=M-m$. The term energy is then given, to the first order in $\beta$, by the sum of the rotational term and of the matrix element (6) with $n=n^{\prime}$ and $m=m^{\prime}$ :

$$
\begin{aligned}
& W_{n, m, m_{I}}=\left(\dot{\hbar}^{2} / 2 A\right)\left[w_{n, m}-\beta^{\prime} d(I)_{m I}^{m_{I}} R_{n, m}^{n, m}\right]= \\
& \left(\dot{\hbar}^{2} / 2 A\right)\left\{w_{n, m}-\beta^{\prime}\left[3 m_{I}^{2}-I(I+1)\right] R(n, m)\right\}= \\
& W_{n, m,-m_{I}}=W_{n,-m, m_{I}}=W_{n,-m,-m_{I}}^{r} .
\end{aligned}
$$

Each rotational energy level of energy $w_{n,|m|}$ splits thus, in this approximation, into $I+\frac{1}{2}$ levels if $I$ is a half-integer, into $I+1$ levels if $I$ is an integer. The absolute values of the intervals between the levels of such a multiplet are functions of the electric field (through $R$ ), but their ratios are independent of it.

Both nuclei have a quadrupole moment.-Each term is now characterized by four quantum numbers $n, m, m_{I 1}, m_{I 2}$. The term energy is, again to the first order in $\beta$ :

$$
\begin{aligned}
& W_{n, \pm m, \pm m_{I 1}, \pm m_{I 2}}= \\
& \left(\hbar^{2} / 2 A\right)\left\{w_{n, m}-\beta_{1}^{\prime}\left[3 m_{I 1}^{2}-I_{1}\left(I_{1}+1\right)\right] R(n, m)-\right. \\
& \left.\beta_{2}^{\prime}\left[3 m_{I 2}^{2}-I_{2}\left(I_{2}+1\right)\right] R(n, m)\right\}
\end{aligned}
$$

IV. The Special Case: $\alpha^{2} \gg|\beta|,|m|=1$

Only one of the nuclei has a quadrupole moment.The energy of terms with $m=1, m_{I}=(I-1)$ or $I$, $M=I$ or $(I+1)$, or with $m=-1, m_{I}=-(I-1)$ or $-I, M=-I$ or $-(I+1)$, is still given by eq 7 . 
For other terms, with $|M|<I, m$ is no longer a good quantum number, but $|m|$ is. The term energy $\epsilon\left(\hbar^{2} / 2 A\right)$ is then obtained, to the first order in $\beta$, by solving the secular equation,

$\left|\begin{array}{ll}w_{n 1}+\left(n, 1\left|H_{Q}\right| n, 1\right)-\epsilon \quad\left(n, 1\left|H_{Q}\right| n,-1\right) & \\ \left.\left(n,-1\left|H_{Q}\right| n, 1\right) \quad w_{n,-1}+(n,-1)\left|H_{Q}\right| n,-1\right)-\epsilon\end{array}\right|=0$,

with $R(n, 1)=R(n,-1), w_{n, 1}=w_{n,-1}$. Its solution is

$$
\begin{gathered}
\epsilon=W_{n, 1, M} /\left(\hbar^{2} / 2 A\right)=W_{n, 1,-M} /\left(\hbar^{2} / 2 A\right)=w_{n 1}-\beta^{\prime} R(n, 1)\left\{3\left(M^{2}+1\right)-I(I+1) \pm\right. \\
\left.\left[(3 / 2)(I+M)(I+M+1)(I-M)(I-M+1) S(n)^{2} / R(n, 1)^{2}+36 M^{2}\right]^{1 / 2}\right\} .
\end{gathered}
$$

The original $w_{n 1}$ rotational level is thus split into $2 I+1$ different levels, if $I$ a half integer, and into $2(I+1)$, if $l$ is an integer.

Both nuclei have a quadrupole moment.-This case is similar to the preceding one, but more complicated. There are three levels with total magnetic quantum number $|M|=I_{1}+I_{2}$ or $I_{1}+I_{2}+1$, for which the energy can be calculated by means of eq 8. These energies are indicated by the following symbols:

$$
\begin{aligned}
& W_{n, 1, I_{1}, I_{2}}=W_{n,-1,-I_{1},-I_{2}} \\
& W_{n, 1, I_{1}, I_{2}-1}=W_{n,-1,-I_{1},-I_{2}+1} \\
& W_{n, 1, I_{1}-1, I_{2}}=W_{n,-1,-I_{1}+1,-I_{2}}
\end{aligned}
$$

For each group of terms with $|M|<I_{1}+I_{2}$ one must solve two secular equations that in general will have more than two rows and columns. The rows and columns correspond to zero-order states characterized by particular values of $\left(m, m_{I 1}, m_{I 2}\right)$ with $|m|=1, m+m_{I 1}+m_{I 2}=M$. The zero-order states belonging to each secular equation can be arranged in a succession, which (for $M>0, I_{2}>I_{1}$ ) can be of either type:

$$
\begin{aligned}
& \left(1, I_{1}, M-I_{1}-1\right),\left(-1, I_{1}, M-I_{1}+1\right), \\
& \left(1, I_{1}-2, M-I_{1}+1\right),\left(-1, I_{1}-2, M-I_{1}+3\right) \ldots
\end{aligned}
$$

or

$$
\begin{aligned}
& \left(1, I_{1}-1, M-I_{1}\right),\left(-1, I_{1}-1, M-I_{1}+2\right), \\
& \left(1, I_{1}-3, M-I_{1}+2\right),\left(-1, I_{1}-3, M-I_{1}+4\right) \ldots
\end{aligned}
$$

The diagonal elements of the secular equation are of the type:

$$
w_{n 1}-R(n, 1)\left[\beta^{\prime}{ }_{1} d\left(I_{1}\right)_{m_{I 1}}^{m_{I 1}}+\beta^{\prime}{ }_{2} d\left(I_{2}\right)_{m_{I 2}}^{m_{I 2}}\right]-\epsilon ;
$$

the elements next to the diagonal are of the type:

$$
-S(n) \beta^{\prime}{ }_{1} d\left(I_{1}\right)_{m_{I 1}}^{m_{I 1}+2} \text { or }-S(n) \beta^{\prime}{ }_{2} d\left(I_{2}\right)_{m_{I 2}}^{m_{I 2}-2}
$$

as the case may be; all other elements vanish.

\section{The Special Case: $\alpha \ll 1, n>0^{5}$}

Only one nucleus has a quadrupole moment.For $\alpha \ll 1$, or, more accurately, for:

$$
\alpha^{2} / 2 n(n+1) \sim\left[\alpha /\left(w_{n+1, m}-w_{n, m}\right)\right]^{2} \lesssim|\beta| \ll 1
$$

the difference $R_{n m}^{n m^{\prime}}-D_{n m}^{n m^{\prime}} \sim \alpha^{2}$ can be disregarded, since $R$ is multiplied by $\beta$ in the expression of the matrix elements of quadrupole interaction.

Replacing then $R$ with $D$ and using the approximate expression for $w_{n, m}$ valid for small $\alpha^{6}$, the matrix element of the complete energy operator pertaining to states with the same values of $n, I$, and $M=m+m_{I}$ is

$$
\begin{gathered}
\left(m^{\prime}|H| m\right)=\left(\hbar^{2} / 2 A\right)\left\{n(n+1) \delta_{m, m^{\prime}}-\right. \\
\beta(-1)^{m \prime-m} D_{n m}^{n m^{\prime}}\left[d(I)_{M-m}^{M-m^{\prime}} / I(2 I-1)-\delta_{m . m^{\prime}} \alpha^{2} / 2 n(n+1) \beta\right]+ \\
\text { terms in } \alpha^{4}, \alpha^{2} \beta \text {, or } \beta^{2}, \text { etc. }
\end{gathered}
$$

The term energy $\epsilon\left(h^{2} / 2 A\right)$ is then obtained, to the first order in $\beta$, by solving the secular equation:

$$
\left|\left(m^{\prime}|H| m\right)-\epsilon\left(\hbar^{2} / 2 A\right) \delta_{m, m^{\prime}}\right|=0 .
$$

The number of rows and columns in the determinant, and hence the degree of the equation, is

\footnotetext{
- For $\alpha \ll 1, n=0$ we have $R_{00}^{00} \approx \alpha^{2}$; hence the quadrupole interaction can be disregarded.

${ }_{6}$ This expression can be given in terms of the coefficient $D_{n m}^{n m}$, provided $n>0$.
}

$\leqslant(2 n+1)$ or $\leqslant(2 I+1)$, whichever is the smaller, since $m$ and $m^{\prime}$ take all the values that do not exceed either $(M+I)$ or $n$ and are not less than either $(M-I)$ or $-n$. The term energy can also be indicated as:

$$
\left(\hbar^{2} / 2 A\right)\left[\left(n(n+1)+\beta^{\prime \prime} y\right)\right],
$$

where $\beta^{\prime \prime}=\beta /(2 n-1)(2 n+3) I(21-1)$ and $y$ is a solution of the secular equation 
$\left|(-1)^{m-m^{\prime}} d(n)_{m}^{m^{\prime}}\left(d(I) \stackrel{M-m^{\prime}}{M-m}+\delta_{m^{\prime}, m} x\right)-\delta_{m^{\prime}, m} y\right|=0$

Here $x=-[I(2 I-1) / 2 n(n+1)]\left(\alpha^{2} / \beta\right)$. The coefficients $d$ are given in table 2 , hence the further steps in calculating $y$ for any given value of $x$ (i. e., of the field strength) are straightforward, even though they may be laborious. The particular case for $n=1$ is worked out in the appendix, which also contains some comments about the methods of solving the secular equation.

Both nuclei have quadrupole moments.- The calculation is similar to that of the preceding case but is more complicated. The energy of each term can be expressed as:

$$
\left(\hbar^{2} / 2 A\right)[n(n+1)+y \bar{\beta} /(2 n-1)(2 n+3)] .
$$

Where $y$ is a solution of the secular equation:

$$
\left|\begin{array}{l}
(-1)^{m^{\prime}-m} d(n)_{m}^{m^{\prime}}\left[b_{1} d_{1}\left(I_{1}\right)_{m_{I 1}}^{m_{I 1}^{\prime}} \delta_{m_{I 2}^{\prime}, m_{I 2}}+b_{2} d\left(I_{2}\right)_{m_{I 2} m_{2}^{\prime}}^{m_{I_{2}}^{\prime}} \delta_{m_{I 1}^{\prime}, m_{I 1}}+\right. \\
\left.x \delta_{m_{I 1}^{\prime}, m_{I 1}} \delta_{m_{I 2}^{\prime}, m_{I 2}}\right]-y \delta_{m_{I 1}^{\prime}, m_{I 1} \delta_{m_{I 2}, m_{I 2}}}
\end{array}\right|=0 .
$$

Here $x=-\alpha^{2} / \bar{\beta} 2 n(n+1)$ and $\bar{\beta}, b_{1}$ and $b_{2}$ are so chosen that:

$$
\bar{\beta} b_{1}=\beta_{1} / I_{1}\left(2 I_{1}-1\right), \bar{\beta} b_{2}=\beta_{2} / I_{2}\left(2 I_{2}-1\right), b_{1} \sim b_{2} \sim 1 .
$$

Finally, $|m|,\left|m^{\prime}\right| \leqslant n ;\left|m_{I 1}\right|,\left|m_{I 1}^{\prime}\right| \leqslant I_{1} ;\left|m_{I 2}\right|,\left|m^{\prime}{ }_{I 2}\right| \leqslant I_{2}$; $m+m_{I 1}+m_{I 2}=m^{\prime}+m_{I 1}^{\prime}+m_{I 2}^{\prime}=M$.

\section{The Special Case: $\alpha^{2} \ll|\beta|$}

Zero field. Only one nucleus has a quadrupole moment.-In this case the total angular momentum $\boldsymbol{F}=\boldsymbol{J}+\boldsymbol{I}(J=n)$, i. e., the sum of the rotational angular momentum and of the spin of the nucleus having a quadrupole moment, is a constant of the motion of the system. $\boldsymbol{J}$ and $\boldsymbol{I}$ are not constant but precess freely around $\boldsymbol{F}$ on account of the quadrupole interaction. All the states having the same value of $F$ and different values of the total magnetic quantum number $M=m+m_{T}$ have the same energy. As shown by Casimir [4] ${ }^{7}$ the energy of each term is proportional to an eigenvalue of the quadrupole coupling operator:

$$
\frac{3}{2}(\boldsymbol{J} \cdot \boldsymbol{I})(\boldsymbol{J} \cdot \boldsymbol{I})+\frac{3}{4}(\boldsymbol{J} \cdot \boldsymbol{I})-\frac{1}{2}(\boldsymbol{J} \cdot \boldsymbol{J})(\boldsymbol{I} \cdot \boldsymbol{I}) .
$$

The proportionality factor can be determined by comparison with the solution obtained in section $\mathrm{V}$ $\mathrm{f}_{\text {or }}$ the case $M=F=n+I, a^{2}=0$, which is trivial.

The energy levels for $n+I \geqslant F \geqslant|n-I|$ are

$\left(\hbar^{2} / 2 A\{n(n+1)+\beta[3 C(C+1)-4 n(n+1) I(I+1)]\right.$ $/ 2(2 n-1)(2 n+3) I(2 I-1)\}$,

with

$$
C=2(\boldsymbol{J} \cdot \boldsymbol{I})=F(F+1)-n(n+1)-I(I+1) .
$$

Near-zero field. Only one nucleus has a quadrupole moment.-The weak electric field has the effect of splitting the levels with equal $F$ and different $M$. The energy is calculated by considering the term proportional to $\alpha^{2}$ in eq 10 , that is,

$$
\alpha^{2} D_{n m}^{n m} / 2 n(n+1),
$$

as a small perturbation. This means simply averaging the value of this term over the participation of each rotational state $(n, m)$ in the complete state $(n, F, M)$. The following term must therefore be added to eq 12 :

$$
\begin{gathered}
\left(\bar{\hbar}^{2} / 2 A\right)\left[\alpha^{2} / 2 n(n+1)\right] \Sigma_{m}\left(C_{F, m, M-m}^{(n I)}\right)^{2} D_{n m}^{n m}=-\alpha^{2}\left(\hbar^{2} / 2 A\right)\left[3 M^{2}-F(F+1)\right][3 D(D-1)-4 F(F+1) n(n+1)] / \\
2 n(n+1)(2 n-1)(2 n+3) 2 F(F+1)(2 F-1)(2 F+3),
\end{gathered}
$$

where $C_{F, m, M-m}^{(n I)}$ is the coefficient of the eigenfunction $(n, m ; I, M-m)$ in the linear combination which constitutes the eigenfunction $(n, F, M)$ [5], and

$$
D=2(\boldsymbol{J} \cdot \boldsymbol{F})=F(F+1)+n(n+1)-I(I+1) .
$$

\footnotetext{
${ }^{7}$ H. B. G. Casimir, Archives du Mus e Teyler [III] VIII, 201 (1936). The application to a molecule in zero-field has been made by B. T. Feld and W. E. Lamb, Jr. (Phys. Rev. 6\%, 15 (1945)). The definition of $q$ by Feid and Lamb is, however, different from that introduced in this paper; this entails a difference in the multiplieative factor of some formulas.
}

In the theory of the anomalous Zeeman effect [6] for a case of $(L S)$ coupling, which is somewhat analagous to the present calculation, one has to calculate the average value of the quantum numbers $m_{L}$ and $m_{S}$, namely, $\Sigma_{m_{L}}\left(C_{J, m_{L}, M-m_{L}}^{(\mathrm{LS})}\right)^{2} m_{L}$, etc. It is shown by direct methods, however, that the results are $M(\boldsymbol{L} \cdot \boldsymbol{J}) / \boldsymbol{J}(\boldsymbol{J}+1)$ and $M(\boldsymbol{S} \cdot \boldsymbol{J}) / \boldsymbol{J}(\boldsymbol{J}+1)$. In our case the quantity $D_{n m}^{n m}$ is a matrix element of quadrupole interaction, 
while $m_{L}$ is a matrix element of dipole interaction. It might therefore be expected that eq 13 could be expressed directly by means of quadrupole matrix elements. In fact eq 13 involves the expression $D_{F M}^{F M}$ and the matrix element of the operator:

$$
\frac{3}{2}(\boldsymbol{J} \cdot \boldsymbol{F})(\boldsymbol{J} \cdot \boldsymbol{F})-\frac{3}{4}(\boldsymbol{J} \cdot \boldsymbol{F})-\frac{1}{2}(\boldsymbol{J} \cdot \boldsymbol{J})(\boldsymbol{F} \cdot \boldsymbol{F}),
$$

but the latter differs from the usual quadrupole coupling operator by the sign of the second term.

Both nuclei have quadrupole moments. - This case is again similar to the preceding ones but more complicated. Equation 13 should be extended to include two terms, one involving the coupling of $\boldsymbol{J}$ and $\boldsymbol{I}_{1}$, and the other that of $\boldsymbol{J}$ and $\boldsymbol{I}_{2}$. However, neither $\boldsymbol{J}+\boldsymbol{I}_{1}$ nor $\boldsymbol{J}+\boldsymbol{I}_{2}$ is a constant of the motion, but only the sum $\boldsymbol{F}=\boldsymbol{J}+\boldsymbol{I}_{1}+\boldsymbol{I}_{2}$. In general, there will be more than one term with the same values of $n$ and $F$. The characterization of each term and the evaluation of $\left(\boldsymbol{J} \cdot \boldsymbol{I}_{1}\right)$ and $\left(\boldsymbol{J} \cdot \boldsymbol{I}_{2}\right)$, and hence of the energy levels, depend upon the ratio $\beta_{1} / \beta_{2}$ and require the application of methods similar to those used in the theory of complex atomic spectra involving a number of terms with the same angular momentum [6, p. 233]. The same holds for the extension of equation 13 .

The author is indebted to Prof. I. I. Rabi and his associates for suggesting this work, for friendly discussions, and for hospitality in the Pupin Physics Laboratory.

\section{References}

[1] F. Brouwer, Utrecht Dissertation (1930).

[2] I. I. Rabi, S. Millman, P. Kusch, and J. R. Zacharias, Phys. Rev. 55, 526 (1939).

[3] H. K. Hughes, Phys. Rev. 70, 570 (1946); 72, 614 (1947).

[4] H. B. G. Casimir, Physica 2, 719 (1935).

[5] E. Wigner, Gruppentheorie, p. 206 (Vieweg, Braunschweig, 1931).

[6] E. U. Condon and G. H. Shortley, Theory of Atomic spectra, p. 380 (Cambridge Univ. Press, Cambridge, England, 1935)

\section{Appendix}

The solution of the secular equation $10 \mathrm{a}$ for $n=1$ is obtained as follows. The total azimuthal quantum number $M$ assumes all the values $|M| \leqslant I+1$. For $|M|=I+1$ the secular equation has only onē row and column; its trivial solution is:

$$
y=I(2 I-1)+x, \text { for }|M|=I+1 .
$$

For $|M|=I$, the equation is quadratic:

$$
\left|\begin{array}{cc}
{[(I-3)(2 I-1)+x]-y} & 3(2 I-1) I^{1 / 2} \\
3(2 I-1) I^{1 / 2} & -2[I(2 I-1)+x]-y
\end{array}\right|=0,
$$

and its solution is

$y=-\frac{1}{2}\{(I+3)(2 I-1)+x \pm$

$\left.3\left([(I-1)(2 I-1)+x]^{2}+4[I(2 I-1)]^{2}\right)^{1 / 2}\right\}$, for $|M|=I$.

For $|M|<I$, the equation is cubic, having three rows and columns. Its solution can be indicated as

$$
y=K_{1}+2 K_{2}^{1 / 2} \cos [(\alpha+2 p \pi) / 3] \text {, for }|M|<I,
$$

where $p=0,1,2, \cos \alpha=-K_{3} / K_{2}^{3 / 2} . \quad K_{1}, K_{2}, K_{3}$ are polynomials of first, second, and third degree in the elements of the determinant form of the secular equation, which can be given in terms of $I, M$ and $x$ :

$$
\begin{aligned}
K_{1} & =2 \\
K_{2} & =\left[4 I^{2}(I+1)^{2}+I(I+1)+1\right]+2\left[3 M^{2}-I(I+1)+1\right] x+x^{2} \\
K_{3} & =\left[8 I^{3}(I+1)^{3}-24 I^{2}(I+1)^{2}+(3 / 2) I(I+1)\right]- \\
3 & \left\{3 M^{2}[2 I(I+1)-7 / 2]-\frac{1}{2} I(I+1)[4 I(I+1)+1]+1\right\} x+ \\
3 & {\left[3 M^{2}-I(I+1)+1\right] x^{2}+x^{3} . }
\end{aligned}
$$

Figure 1 shows the plot of $y$ as a function of $x$ for $I=7 / 2$, corresponding to the $\mathrm{Cs}$ nucleus in the $\mathrm{CsF}$ molecule ( $\mathrm{F}$ has no quadrupole moment), and for all values of $M$. The position of the energy levels for $x$ greater than two or three times $I(2 I-1)$ is already given with good accuracy by eq 7 and 9 , which were calculated on the assumption of $\alpha^{2} \gg|\beta|$.

The derivation of the eq 15 is a purely algebraic but rather tedious and laborious process. This is probably true in general of the process of solving secular equations in which the matrix element are still functions of various pertinent parameters. It is not just the solution of the equation that is tedious, but especially the preliminary process of transforming the equation from its determinant form to a polynomial form. The labor involved increases rapidly with the degree of the equation. Therefore, whenever it is necessary to obtain a solution only for a few values of the parameters $I$ and $M$ and $x$, it is advisable to introduce these values in the matrix elements themselves, and to carry out the rest of the work numerically. Otherwise it may be advisable to consider other ways of reducing the equation to a polynomial form. For example, the coefficients of an algebraic equation can be expressed in terms of the sums of powers of its unknown solutions $y_{i}$. In the example above, $K_{1}=S_{1} / 3, \quad K_{2}=\left(S_{2}-S_{1}^{2} / 3\right) / 6$, $K_{3}=\left(\left(S_{3}-S_{1} S_{2}\right) / 6\right)-S_{1}^{3} / 27$, where $S_{n}=\Sigma_{i} y_{i}^{n}$. The quantity $S_{n}$ is the trace of the matrix of the $n$-th power of the energy operator; it may well be simpler and safer from mistakes to calculate the necessary $S_{n}$ than to expand the determinant form of the secular equation. 

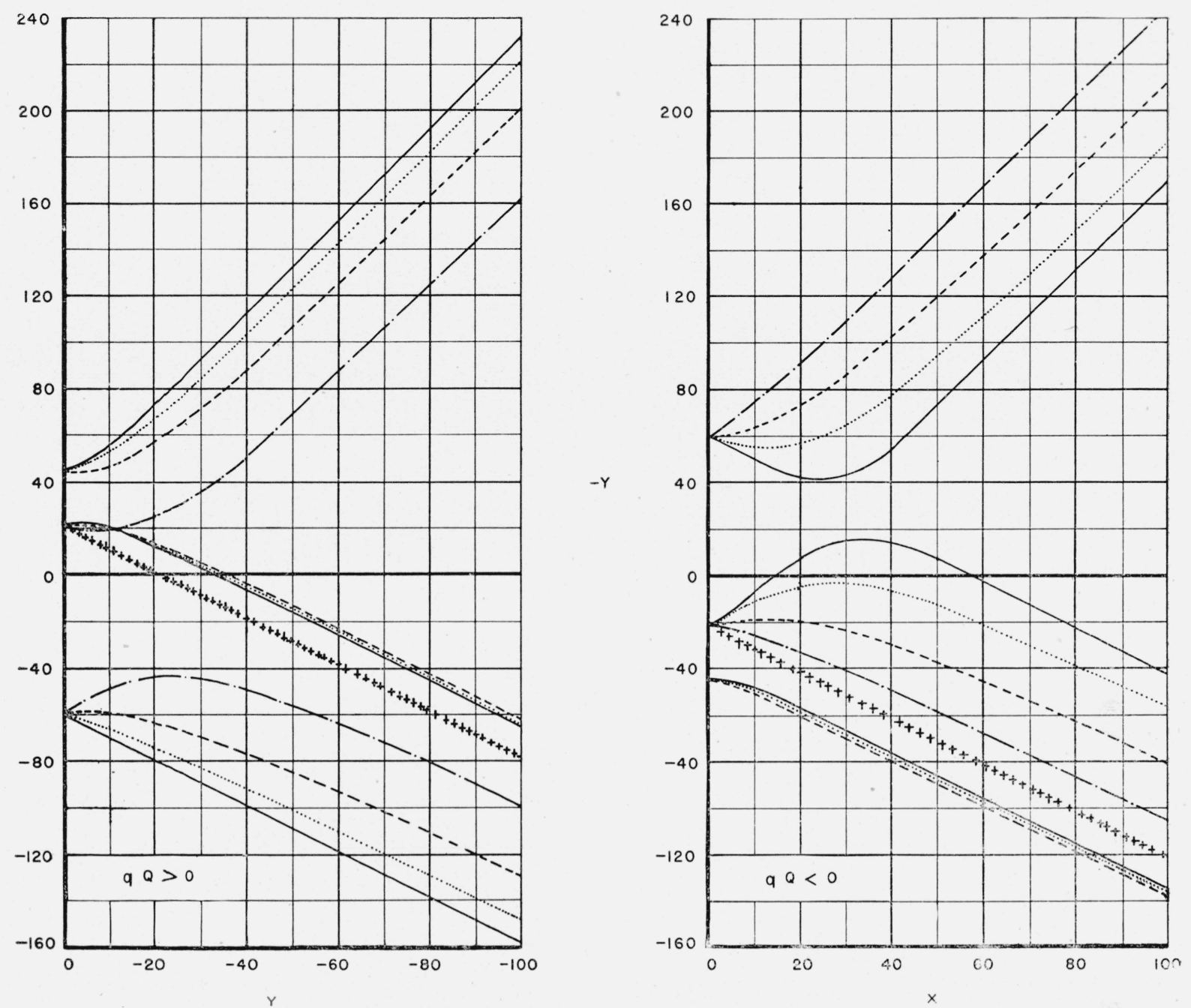

FIGURE 1.-Plot of the energy levels $W_{n}$ versus the square of the electric field, $E^{2}$, for $n=1, I=7 / 2,|M| \leq I+1=9 / 2 ;$ coordinates are in dimensionless units:

$x=-21(l E)^{2} / e^{2} q Q\left(h^{2} / 2 A\right), y=420 W / e^{2} q Q$.

,$M= \pm 1 / 2 ; \ldots, M= \pm 3 / 2 ;--\longrightarrow, M= \pm 5 / 2 ;-\cdots \cdots, M= \pm 7 / 2 ;++++, M= \pm 9 / 2$.

Washington, March 31, 1947. 